\title{
Efeito da administração de uma dieta enteral com antioxidantes sobre as concentrações plasmáticas de tióis totais, carbonilas de proteínas e malondialdeído após acidente vascular cerebral
}

\author{
Effect of a commercial enteral diet with added \\ antioxidants on total plasma thiol, protein \\ carbonyl and malondialdehyde \\ levels after a stroke
}

\author{
Lorene Simioni YASSIN ${ }^{1}$ \\ Chika FUKUI $^{1}$ \\ Pamela Cristiani Dias PEREIRA ${ }^{1}$ \\ Marcia OLANDOSKI ${ }^{2}$ \\ Paulo Roberto AVELES ${ }^{2}$ \\ Silvia Carolina PINTO2 \\ Caroline RESNAUER ${ }^{1}$ \\ Lia Sumie NAKAO ${ }^{2}$ \\ Ivone Ikeda MORIMOTO3
}

\section{RE S U M O}

\section{Objetivo}

Avaliar o efeito da administração de uma dieta enteral industrializada com antioxidantes sobre as concentrações plasmáticas de tióis totais, carbonilas de proteínas e malondialdeído em pacientes após acidente vascular cerebral.

\section{Métodos}

A amostra foi constituída de 14 pacientes de um hospital geral que iniciaram nutrição enteral 48 horas após o evento. Falência múltipla, insuficiência hepática, obesidade mórbida e diabetes Mellitus associados foram

\footnotetext{
${ }^{1}$ Nutricionistas. Curitiba, PR, Brasil.

2 Pontifícia Universidade Católica do Paraná, Pós-Graduação em Ciências da Saúde. Curitiba, PR, Brasil.

3 Pontifícia Universidade Católica do Paraná, Curso de Nutrição. R. Imaculada Conceição, 1155, 80215-901, Curitiba, PR, Brasil. Correspondência para/Correspondence to: II MORIMOTO. E-mail: <ivone.morimoto@pucpr.br>.
} 
critérios de exclusão. A dieta industrializada ofertada por gotejamento contínuo, com uso de bombas infusoras, continha mix de carotenoides, vitaminas C, E e minerais Se, Zn e Cu em sua formulação. As amostras de sangue foram coletadas antes do início da administração da dieta e após cinco dias de início da dieta enteral, somente de pacientes que tivessem recebido o volume necessário para completar o gasto energético total. Tióis plasmáticos e carbonilas de proteína foram determinados por meio do Reagente de Ellman e pela reação com dinitrofenilhidrazina respectivamente. O malondialdeído foi obtido pela determinação de substâncias reativas do ácido tiobarbitúrico.

\section{Resultados}

A média de idade foi $M=70,3, D P=14,1$ anos. Todos receberam acima de $100 \%$ da Dietary Reference Intakes para nutrientes antioxidantes, que não ultrapassaram os limites superiores toleráveis de ingestão. Não houve alteração da concentração de tióis, mas houve aumento da formação de carbonilas de proteínas $(p=0,034)$. Nos pacientes entubados, esse marcador mostrou-se significativamente maior $(p=0,048)$ após administração da dieta. Não houve diferença nas concentrações de malondialdeído após a oferta de antioxidantes dietéticos.

\section{Conclusão}

A análise de biomarcadores não demonstrou redução do estresse oxidativo após administração de dieta enteral industrializada com antioxidantes.

Termos de indexação: Acidente cerebrovascular. Estresse carbonílico. Peroxidação de lipídeos. Suplementação alimentar. Terapia nutricional.

\section{A B S T R A C T}

\section{Objective}

The aim of this study was to assess the effect of a commercial enteral diet with added antioxidants on total plasma thiol, protein carbonyl and malondialdehyde levels of stroke survivors.

\section{Methods}

Fourteen patients from a general hospital who had been started on enteral nutrition 48 hours after a stroke were included in the study. The exclusion criteria were multiple organ dysfunction syndrome, liver failure and morbid obesity associated with diabetes Mellitus. The commercial diet was fed by continuous drip via infusion pump and contained mixed carotenoids, vitamins $C$ and $E$, and the minerals selenium, zinc and copper. Blood samples were collected at baseline and after 5 days of enteral diet, but only from patients whose diet intake met their total energy expenditure. Total plasma thiol and protein carbonyl levels were determined by Ellman's reagent and dinitrophenylhydrazine, respectively. Plasma malondialdehyde levels were measured by the assay of thiobarbituric acid reactive substances.

\section{Results}

The mean age of the sample was $M=70.3$ years, $(S D=14.1)$. All patients received more than $100 \%$ of the Dietary Reference Intakes for the abovementioned antioxidants but none exceeded the tolerable upper limit. Plasma thiol and malondialdehyde levels did not vary over time but protein carbonyl levels were significantly higher $(p=0.034)$, especially in intubated patients $(p=0.048)$.

\section{Conclusion}

Biomarker determinations showed that a commercial enteral diet with added antioxidants did not reduce oxidative stress.

Indexing terms: Cerebrovascular accident. Carbonyl stress. Lipid peroxidation. Supplementary feeding. Nutrition therapy.

\section{N T R O D U ÇÃ O}

De acordo com dados do Ministério da Saúde (MS) e da Organização Panamericana de Saúde (OPAS) ${ }^{1}$, no ranking das dez causas de mor- te por fatores conhecidos no Brasil, as doenças cerebrovasculares ocupam o primeiro lugar ${ }^{1}$. Dentre elas, o Acidente Vascular Cerebral (AVC), atualmente denominado Acidente Vascular Encefálico (AVE), apresenta alta prevalência. 
O processo de isquemia e de reperfusão resultante do AVE induz ao estresse oxidativo². No tecido isquêmico, a xantina desidrogenase é convertida em xantina oxidase. Essa enzima atua na formação de superóxido, gerador de outros oxidantes que produzem danos às membranas celulares, ao DNA e às proteínas ${ }^{3}$. Na reoxigenação do tecido isquêmico há elevação das concentrações de espécies reativas em virtude da maior captação de cálcio nas células anóxicas, o que aumenta a produção de xantina oxidase $e^{4,5}$.

A membrana celular cerebral é muito rica em ácidos graxos poli-insaturados e pobre em enzimas antioxidantes e vitamina $\mathrm{E}$, embora disponha de alguma reserva de vitamina $\mathrm{C}^{3}$. Como consequência, os ácidos graxos poli-insaturados da membrana das células cerebrais são sensíveis ao ataque de espécies reativas ${ }^{4}$. 0 dano aos lipídeos, que é mediado por espécies reativas, induz ao fenômeno conhecido como peroxidação lipídica. Um dos produtos da peroxidação lipídica é o Malondialdeído (MDA) ${ }^{4,6}$.

Nas proteínas, os tióis estão presentes nos resíduos de cisteína e são considerados os principais responsáveis pela ação antioxidante de proteínas plasmáticas. Esses resíduos são geralmente reativos e suscetíveis à oxidação, o que origina dissulfetos e ácido sulfônico, formas que inativam a função proteica; portanto, em situações de estresse oxidativo, os níveis de tióis estão reduzidos no plasma ${ }^{7}$. A oxidação proteica pode, também, resultar na formação de compostos carbonila, como o ácido carboxílico ou aldeídos, com igual número de carbonos ou um carbono a menos que o aminoácido de origem. Assim, há aumento das quantidades desse marcador no plasma de indivíduos submetidos a estresse oxidativo ${ }^{7}$.

O sistema antioxidante primário é constituído por enzimas endógenas (superóxido dismutase, catalase e glutationa peroxidase), que têm a capacidade de inibir, até um limite, a gênese ou a toxicidade de espécies reativas ${ }^{8}$. O sistema secundário é formado por substâncias antioxidantes exógenas: os minerais cobre (Cu) zinco (Zn), selênio (Se) e as vitaminas $A, C$ e $E^{8}$. Os minerais cita- dos são componentes das enzimas antioxidantes glutationa peroxidase (GSHPx) e Superóxido Dismutase (SOD). Níveis baixos de $\mathrm{SOD}^{9}$ e redução do selênio plasmático ${ }^{3}$ foram observados nos pacientes, após isquemia cerebral aguda. Adequados níveis de vitamina $E$, vitamina $A$ e licopeno estão associados à redução do risco de microangiopatia relacionada com o dano cerebral ${ }^{9}$. Foi documentado que o tratamento com $\alpha$-tocoferol reduz o nível de apoptose após a isquemia ${ }^{10}$ : esses dados demonstram a importância da nutrição precoce após o AVE.

Os efeitos neurológicos de um AVE são determinados pela área do cérebro afetada ${ }^{11}$. As possíveis manifestações clínicas relacionadas ao prejuízo cognitivo e vascular incluem perda da memória e confusão mental. Além disso, podem resultar em afasia, apraxia, agnosia, perda do controle motor, paralisia, demência, cegueira e disfagia neurogênica ${ }^{11,12}$. Diante dessas complicações, faz-se necessária a utilização da nutrição enteral como via de alimentação. Foi demonstrado que o início do fornecimento de nutrientes para esses pacientes até 72 horas após o evento cerebrovascular reduz a permanência hospitalar ${ }^{13}$.

Embora seja reconhecido o prejuízo subsequente ao AVE produzido pela ação de espécies reativas, não foram encontrados estudos conclusivos sobre o desempenho de vitaminas e minerais antioxidantes ofertados por via enteral na redução ou melhoria do estresse oxidativo. Justifica-se, portanto, a realização deste estudo, que avaliou o efeito da administração de uma dieta enteral industrializada com antioxidantes sobre as concentrações plasmáticas de tióis totais, carbonilas de proteínas e malondialdeído, após acidente vascular cerebral.

\section{MÉTODOS}

Estudo longitudinal realizado em um hospital geral da cidade de Curitiba. Foram incluídos no estudo pacientes admitidos até 48 horas após $A V E$, que permaneceram em nutrição enteral por pelo menos 5 dias, que receberam o volume pre- 
conizado para completar o Gasto Energético Total (GET), calculado até 72 horas e cujos familiares assinaram o termo de consentimento livre e esclarecido. Falência múltipla de órgãos, insuficiência hepática, obesidade mórbida e diabetes Mellitus associados ao AVE foram critérios de exclusão. Este estudo foi aprovado pelo Comitê de Ética em Pesquisa da Pontifícia Universidade Católica do Paraná (PUCPR), protocolo número 404 de 6 de outubro de 2004.

Os participantes receberam dieta enteral com antioxidantes dentro das primeiras 48 horas após o AVE. A fórmula foi administrada por sonda nasogástrica, nasoentérica, gastrostomia ou jejunostomia, de acordo com a indicação da equipe de suporte nutricional do hospital, por gotejamento contínuo, com uso de bombas infusoras. A medida da altura do joelho foi realizada para estimar a altura do paciente. O Índice de Massa Corporal (IMC) médio $\left(20,7 \mathrm{~kg} / \mathrm{m}^{2}\right)$ foi utilizado para obtenção do peso ideal. Esses dados foram utilizados para o cálculo do Gasto Energético Basal (GEB): aplicou-se a fórmula de Harris-Benedict ${ }^{14}$, e o valor obtido foi multiplicado pelo fator injúria ${ }^{15}$, resultando no GET de cada paciente. A partir disso, foi calculado o volume necessário para atingir o GET e a taxa de administração da dieta.

Foi administrada a fórmula padrão Hiper Diet Standard ${ }^{\circledR}$ do laboratório Support de densidade energética $1 \mathrm{kcal} / \mathrm{mL}$, composta de $16 \%$ de proteína, 49\% de carboidrato e 35\% de lipídeo, e que apresenta uma mistura de antioxidantes constituída de $\alpha$, $\beta$ e $\gamma$ caroteno, licopeno, luteína e zeaxantina além dos micronutrientes vitaminas C (100mg/L), E (13mg/L), Se (57mcg/L), Zn (12mg/L) e Cu $(1,7 \mathrm{mg} / \mathrm{L})$.

A primeira coleta de $3 \mathrm{~mL}$ de sangue foi realizada com o paciente em jejum, antes do início da administração da dieta e até 48 horas após o evento. O gotejamento inicial foi de $30 \mathrm{~mL} / \mathrm{hora}$, evoluindo gradualmente $(20 \mathrm{~mL} / \mathrm{hora} / \mathrm{dia})$ até atingir o volume final necessário para totalizar o GET dentro de no máximo 72 horas. Dois dias após a totalização do GET, os pacientes foram submetidos a jejum de 8 horas para coleta de sangue. Assim, a segunda coleta de sangue foi realizada cinco dias após início da administração da dieta. A coleta de amostras de sangue foi realizada por funcionários do laboratório de análises clínicas do hospital. O plasma foi centrifugado e separado em três tubos de ensaio com EDTA. Após esse processo, o plasma foi congelado a menos $80^{\circ} \mathrm{C}$.

As dosagens de carbonilas proteicas e tióis plasmáticos foram realizadas no Laboratório de Bioquímica da PUCPR. A determinação de carbonilas foi realizada pelo método da dinitrofenilhidrazina (DNPH). Em um tubo de ensaio foram colocados $200 \mu \mathrm{L}$ de plasma e $1 \mathrm{~mL}$ de DNPH (10mM em $2 \mathrm{M} \mathrm{HCl}$ ); em outro tubo, o controle foi feito com $200 \mu \mathrm{L}$ de plasma e $1 \mathrm{~mL}$ de $\mathrm{HCl} 2 \mathrm{M}$. Após homogeneizado, foi aquecido em banho-maria a $37^{\circ} \mathrm{C}$ por 90 minutos. As amostras foram resfriadas por 10 minutos em gelo e adicionadas a $1 \mathrm{~mL}$ de Ácido Tricloro Acético (TCA) 28\% m/v. Após homogeneizadas novamente em vórtex por 3 min e centrifugadas a 6000rpm por 3 minutos, foram descartados os sobrenatantes. Na sequência, foi adicionado $1 \mathrm{~mL}$ de etanol - acetato de etila 1:1, homogeneizado em vórtex por $2 \mathrm{~min}$ e centrifugado a $6000 \mathrm{rpm}$ por duas vezes. Ao precipitado acrescentou-se $1 \mathrm{~mL}$ de guanidina $6 \mathrm{M}$. Essa mistura foi homogeneizada durante um minuto, transferida para um microtubo e centrifugado a 6000rpm por 3 minutos. A leitura das amostras foi realizada em espectrofotômetro Biospectro ${ }^{\circledR}$ modelo SP220, em 360nm. O cálculo da concentração de carbonilas utilizou o $\varepsilon=21000 \mathrm{M}^{-1} \cdot \mathrm{cm}^{-1}$, normalizada pela concentração de albumina do paciente e expressa em nmol/mg de proteína. A dosagem de tióis plasmáticos foi por meio do Reagente de Ellman, descrito anteriormente na literatura ${ }^{16}$. A leitura das absorbâncias foi realizada em $405 \mathrm{~nm}$, e as concentrações de tióis totais das amostras foram calculadas e corrigidas pelo valor de albumina, sendo expressas em $\mu \mathrm{mol} / \mathrm{L}$. A dosagem de MDA foi realizada pela equipe do Laboratório de Análises Clínicas da Faculdade de Medicina de Ribeirão Preto da Universidade de São Paulo pelo procedimento descrito por Buege \& Aust ${ }^{17}$. O MDA 
reagiu com o ácido tiobarbitúrico (TBARS) para formar um complexo com absorbância máxima a $535 \mathrm{~nm}$. A quantidade de substâncias que sofreram peroxidação lipídica foi expressa em $\mathrm{nmol} / \mathrm{mL}$ de MDA.

A análise estatística foi realizada com o auxílio do programa BioEstast 2.0. Os resultados dos níveis de tiol e de carbonila foram expressos por médias, desvios-padrões, medianas, valores mínimos e máximos. Para a comparação dos resultados obtidos antes e depois da aplicação da dieta, foi usado o teste $t$ Student para amostras pareadas ou o teste não paramétrico de Wilcoxon, quando apropriado. A condição de normalidade das variáveis foi avaliada pelo teste de Shapiro-Wilks. Para avaliação da correlação entre idade e níveis de tiol e de carbonila, foi estimado o coeficiente de correlação de Pearson. Em todos os testes, valores de $p<0,05$ indicaram significância estatística.

\section{RESULTADOSE DISCUSSÃO}

Foram estudados 24 pacientes com diagnóstico médico de AVE com indicação de terapia nutricional enteral padrão. Destes, 10 evoluíram para o óbito antes da realização da segunda coleta de sangue, sendo o estudo finalizado com 14 pacientes. Bamford et al. ${ }^{18}$ afirmam que 10\% das vítimas do AVE morrem em até 30 dias após o evento. As características gerais dos pacientes que foram a óbito e dos que sobreviveram estão descritas na Tabela 1. Os diagnósticos associados ao AVE foram agrupados segundo as doenças. Dentre os pacientes com doença cardiovascular, predominaram pacientes hipertensos $(87,5 \%)$. Outros diagnósticos associados foram crises convulsivas $(16,7 \%)$, gastrite $(12,5 \%)$, pneumonia aspirativa $(8,3 \%)$, sepse $(8,3 \%)$, trauma $(8,3 \%)$, insuficiência cardíaca $(4,2 \%)$, angina pectoris $(4,2 \%)$, insuficiência renal aguda $(4,2 \%)$, insuficiência renal crônica $(4,2 \%)$, infecção do trato urinário (4,2\%), insuficiência respiratória $(4,2 \%)$, doença pulmonar obstrutiva crônica $(4,1 \%)$, mal de Alzheimer $(4,1 \%)$, hidrocefalia $(4,1 \%)$, neoplasia $(4,1 \%)$, litíase biliar $(4,1 \%)$, hipotireoidismo $(4,1 \%)$, reumatismo $(4,1 \%)$ e desnutrição energético-proteica (4,1\%).

A Média (M) de idade de 70,4 anos, Desvio-Padrão (DP) de 14,1 anos foi compatível com achados de outros estudos. Madamanchi et al. ${ }^{19}$ relataram que adultos com mais de 65 anos são respectivamente 2,5 e 4 vezes mais propensos à hipertensão e à doenças coronarianas do que indivíduos entre 40 e 49 anos. Poulet et al. ${ }^{20}$ afirmam que a hipertensão arterial induz a

Tabela 1. Características gerais dos pacientes do estudo. Curitiba (PR), 2008.

\begin{tabular}{|c|c|c|c|}
\hline \multirow{2}{*}{ Variáveis } & Pacientes que foram à óbito & Pacientes que finalizaram o estudo & Total \\
\hline & $n=10$ & $n=14$ & $n=24$ \\
\hline Idade (média, desvio-padrão) & $M=70,5, D P=14,4$ & $M=70,3, D P=14,5$ & $M=70,4, D P=14,1$ \\
\hline \multicolumn{4}{|l|}{$\operatorname{Sexo}(n)$} \\
\hline Masculino & 3 & 6 & 9 \\
\hline Feminino & 7 & 8 & 15 \\
\hline $\operatorname{AVEI}(n)$ & 7 & 12 & 19 \\
\hline $\operatorname{AVEH}(n)$ & 3 & 2 & 5 \\
\hline \multicolumn{4}{|l|}{ Diagnósticos associados ao AVE } \\
\hline Cardiovascular & 9 & 11 & 20 \\
\hline Renal & 2 & 1 & 3 \\
\hline Pulmonar & 1 & 2 & 3 \\
\hline Neurológico & 2 & 4 & 6 \\
\hline Outras & 4 & 5 & 9 \\
\hline
\end{tabular}

AVEl: Acidente Vascular Encefálico Isquêmico; AVEH: Acidente Vascular Encefálico Hemorrágico; M: Média; DP: Desvio-Padrão. 
neurodegeneração, produzida pelo aumento na geração de espécies reativas de oxigênio e nitrogênio.

Neste estudo, 79,2\% dos acidentes foram classificados como AVE isquêmicos: segundo a Sociedade Brasileira de Doenças Cerebrovasculares $^{21}$, 85\% são dessa natureza.

No presente estudo, as concentrações plasmáticas do marcador proteico tiol não diferiram significativamente quando comparados os períodos anterior e posterior à administração da dieta enteral com antioxidantes (Tabela 2).

Chang et al. ${ }^{22}$ investigaram a relação entre AVE, níveis de antioxidantes plasmáticos e produtos do estresse oxidativo em 36 pacientes internados até $24 \mathrm{~h}$ do evento e verificaram que a concentração de tióis totais plasmáticos, constituídos, sobretudo, de grupamentos tiol da albumina, que são suscetíveis ao dano oxidativo, não sofreu modificações significativas quando comparado ao grupo-controle. Segundo Williams ${ }^{23}$, as frações de aminotióis associadas a proteínas plasmáticas não são biologicamente ativas, entretanto a forma livre dessas frações (reduzida e oxidada) pode ter uma função importante na patogênese do AVE. Andersson et al. ${ }^{24}$ afirmam que a função da cisteína, que representa o tiol de baixo peso molecular mais abundante no plasma, constitui um componente do sistema de defesa antioxidante extracelular, portanto, nas situações de aumento do estresse oxidativo, haveria redução de seus níveis, o que não ocorreu neste estudo.

A dosagem de carbonila foi realizada para 12 pacientes, pois duas amostras de sangue apresentavam hiperlipemia, inviabilizando a dosagem. A concentração de carbonila proteica plasmática foi significativamente maior $(p=0,034)$ após a oferta de dieta enteral quando comparada aos níveis anteriores (Tabela 2), demonstrando que a suplementação de antioxidantes alimentares não foi capaz de reduzir o estresse oxidativo proteico durante o período do estudo.

O coeficiente de correlação entre idade e diferença de tiol (Figura 1) antes e depois da administração de antioxidantes dietéticos foi 0,732, com significância estatística $(p=0,016)$. Esse dado sugere que os mais velhos assimilaram mais tióis, ou que tinham níveis basais menores de tióis do que os mais jovens. Outros estudos confirmam que os idosos sofrem mais de deficiência de tióis: há uma relação diretamente proporcional entre estado redox da cisteína/cistina e a idade, pois o estresse oxidativo é um processo associado ao envelhecimento e agravado pela doença ${ }^{25,26}$.

Tabela 2. Níveis médios de tiol, carbonila de proteína e MDA plasmáticos antes e após o tratamento alimentar. Curitiba (PR), 2008.

\begin{tabular}{|c|c|c|c|c|c|c|}
\hline Variável & Média & Mediana & Mínimo & Máximo & Desvio-padrão & Valor de $p$ \\
\hline \multicolumn{7}{|l|}{ Tiol ( $\mu \mathrm{mol} / \mathrm{L})$} \\
\hline Antes & 0,281 & 0,295 & 0,127 & 0,500 & 0,094 & \multirow{3}{*}{$0,944^{a}$} \\
\hline Depois & 0,292 & 0,310 & $-0,025$ & 0,732 & 0,174 & \\
\hline (Diferença) & 0,011 & 0,010 & $-0,178$ & 0,415 & 0,142 & \\
\hline \multicolumn{7}{|c|}{ Carbonila (nmol/mg de proteína) } \\
\hline Antes & 0,227 & 0,126 & 0,021 & 0,905 & 0,251 & \multirow{3}{*}{$0,034^{b}$} \\
\hline Depois & 0,586 & 0,377 & 0,030 & 1,975 & 0,561 & \\
\hline (Diferença) & 0,359 & 0,252 & $-0,252$ & 1,744 & 0,574 & \\
\hline \multicolumn{7}{|c|}{ MDA $(n m o l / m L)^{*}$} \\
\hline Antes & 0,584 & 0,504 & 0,362 & 0,986 & 0,201 & \multirow{3}{*}{$0,975^{\mathbf{b}}$} \\
\hline Depois & 0,578 & 0,499 & 0,309 & 1,259 & 0,260 & \\
\hline (Diferença) & $-0,006$ & 0,034 & 0,760 & 0,760 & 0,317 & \\
\hline
\end{tabular}

a Teste $t$ Student para amostras pareadas, $p<0,05 ;$ ' ${ }^{2}$ Teste não paramétrico de Mann-Whitney, $p<0,05 ;{ }^{*}$ Dosagem realizada no Laboratório de Análises Clínicas da FMRP-USP. 
O coeficiente de correlação entre idade e diferença de carbonila antes e depois da oferta de antioxidantes alimentares (Figura 2 ) resultou em 0,262 , sem significância estatística $(p=0,464)$. Um estudo avaliou o efeito de dieta enteral com antioxidantes na síndrome da insuficiência respiratória aguda e descreveu que, nessa situação, as espécies reativas e mediadores inflamatórios encontram-se aumentados. Atribuiu-se esse resultado possivelmente ao estresse oxidativo produzido pela ventilação mecânica ${ }^{27}$. A ventilação mecânica produz aumento da proteólise na magnitude de $105 \%$. A atividade do proteosomo 20 envolvido no mecanismo de dano celular no

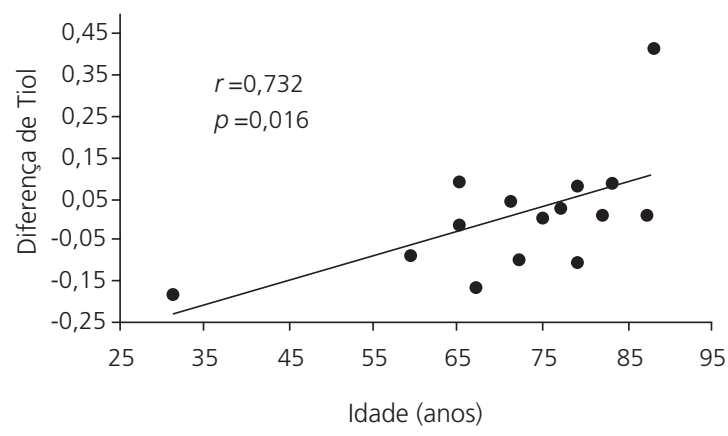

Figura 1. Correlação entre a idade (anos) e a diferença nas concentrações plasmáticasde tiois após administração de dieta enteral com antioxidantes. Curitiba (PR), 2008.

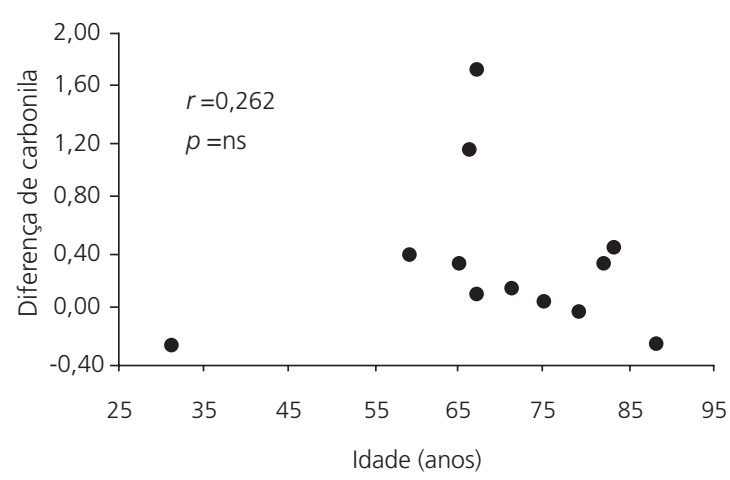

Figura 2. Correlação entre a idade (anos) e a diferença nas concentrações plasmáticas de carbonilas de proteínas após administração de dieta enteral com antioxidantes. Curitiba (PR), 2008. diafragma resultou em valor $76 \%$ maior quando comparado àqueles que não se encontravam em ventilação mecânica ${ }^{28}$. Neste estudo, não houve diferença significativa $(p=0,224)$ para a concentração de tióis, entretanto nas concentrações de carbonila de proteína houve aumento significativo desses valores $(p=0,048)$ após 5 dias de início da oferta de dieta enteral industrializada com antioxidantes nos 8 pacientes que tiveram indicação de ventilação mecânica durante o período do estudo, confirmando a hipótese de que a ventilação mecânica pode aumentar o estresse oxidativo proteico. Zergeroglu et al. ${ }^{29}$ verificaram aumento de carbonilas de proteínas e formação de hidroperóxidos após 6 e 18 horas de ventilação mecânica, indicando injúria oxidativa no diafragma resultante desse tratamento.

A concentração de MDA inicial dos pacientes até 48 horas após o evento foi em média de $0,535, \mathrm{DP}=0,19 \mathrm{nmol} / \mathrm{mL}$. Polidori et al..$^{30}$ encontraram níveis plasmáticos semelhantes ( $M=0,60$, $\mathrm{DP}=0,13 \mu \mathrm{mol} / \mathrm{L}) 24$ horas após AVE isquêmico, porém não houve acompanhamento após início da alimentação. El Kossi \& Zakhary ${ }^{31}$ relataram elevação significativa na peroxidação lipídica 48 horas após o evento $(M=1,71, D P=0,38 \mu \mathrm{mol} / \mathrm{L})$.

Dois estudos ${ }^{32,33}$ avaliaram o MDA e a mortalidade em pacientes críticos, não especificamente no AVE. A elevada concentração de MDA não foi considerada preditor de óbito. No estudo de Preiser et al. ${ }^{32}$, não houve diferença nos valores do MDA e da mortalidade entre os pacientes críticos que receberam intervenção de antioxidantes e os que não receberam. Crimi et al..$^{33}$ verificaram redução significativa nos níveis de TBARS $(p<0,01)$ de 5,33 para $2,42 \mathrm{nmol} / \mathrm{mL}$ e na mortalidade do grupo de pacientes críticos submetidos à suplementação com antioxidantes por via enteral. A mortalidade foi de $45,7 \%$ no grupo que recebeu suplementação e $67,5 \%$ no grupo não suplementado.

Neste estudo, a diferença entre os níveis de MDA em jejum e após a oferta de dieta enteral não foi estatisticamente significativa $(p=0,998)$, demonstrando que não houve redução na peroxi- 
dação lipídica com a administração de antioxidantes alimentares.

Os resultados encontrados conduzem à análise de alguns fatores envolvidos: (a) quantidade de micronutrientes antioxidantes, (b) tempo de suplementação e (c) capacidade antioxidante nas doenças crônicas.

A quantidade de micronutrientes antioxidantes fornecida por meio da dieta enteral foi comparada às recomendações da Dietary Reference Intakes (DRI) ${ }^{34,35}$. Os pacientes receberam em média $1230 \mu \mathrm{g} / \mathrm{dia}$ RE de vitamina $A$ (RDA $=700 \mu \mathrm{g} /$ dia para mulheres e $900 \mu \mathrm{g} /$ dia para homens), sendo 3mg de carotenoides; 19,5mg/ dia de vitamina $\mathrm{E}(\mathrm{RDA}=15 \mathrm{mg} / \mathrm{dia}$ para ambos os sexos), $150 \mathrm{mg} /$ dia de vitamina $C$ (RDA $=75 \mathrm{mg} / \mathrm{dia}$ para mulheres e $90 \mathrm{mg} / \mathrm{dia}$ para homens); $90 \mu \mathrm{g} /$ dia de Se $(\mathrm{RDA}=55 \mu \mathrm{g} /$ dia para mulheres e homens), $18 \mathrm{mg} / \mathrm{dia}$ de $\mathrm{Zn}(\mathrm{RDA}=8 \mathrm{mg} / \mathrm{dia}$ para mulheres e $11 \mathrm{mg} /$ dia para homens) e $2,7 \mathrm{mg} / \mathrm{dia}$ de $\mathrm{Cu}$ ( $\mathrm{RDA}=0,9 \mathrm{mg} /$ dia para mulheres e homens). Verificou-se, portanto, que todos os pacientes receberam quantidades superiores a $100 \%$ da DRI dessas vitaminas e minerais. Essas quantidades não ultrapassaram o limite máximo de ingestão (UL) estabelecido na DRI.

Estudos relataram a redução da peroxidação lipídica após a suplementação de vitaminas antioxidantes em quantidades específicas ${ }^{36,37}$. Crimi et al. ${ }^{33}$, ao suplementarem $500 \mathrm{mg} / \mathrm{d}$ de vitamina $C$ e $400 U l / d$ de vitamina $E$, avaliaram uma redução significativa $(p<0,01)$ nos níveis de MDA, o que melhora a resistência da LDL ao estresse oxidativo em aproximadamente $30 \%$ nos pacientes críticos. Abilés et al. ${ }^{36}$ relataram que pacientes críticos que receberam uma quantidade de vitaminas antioxidantes inferior a $66 \%$ da DRI tiveram um aumento na peroxidação lipídica e que a administração de 66 a 100\% da DRI de vitaminas antioxidantes reduziu o risco de agravar o estresse oxidativo em $94 \%$. No estudo de Ullegaddi et al. ${ }^{37}$, a suplementação via oral de 800 UI de $\alpha$-tocoferol e $500 \mathrm{mg}$ de vitamina $C$ foi capaz de aumentar a concentração plasmática de $\alpha$-tocoferol e de vitamina $C$ além de reduzir signifi- cativamente $(p<0,002)$ a concentração plasmática de MDA no grupo AVE isquêmico com suplementação, em contraste com o aumento constatado no grupo não suplementado. O estudo de Elmadfa et $a / .^{38}$ demonstrou que a suplementação com $40 \mathrm{mg} / \mathrm{dia}$ de beta caroteno reduziu significativamente (16\%) a concentração de MDA. Observou-se que nos estudos citados anteriormente, as doses de vitaminas antioxidantes utilizadas foram superiores às aplicadas neste estudo, porém sem ultrapassar os níveis máximos de ingestão tolerável.

Em contradição, dois estudos - um deles realizado com pacientes críticos ${ }^{33}$ e outro com pacientes saudáveis - nos quais houve suplementação de vitaminas A, C, E não encontraram diferença significativa após a suplementação ${ }^{38}$.

Como não foram encontrados outros estudos sobre o efeito da suplementação de antioxidantes alimentares por via enteral nos valores de marcadores proteicos do estresse oxidativo, a análise quanto ao tempo de suplementação foi realizada somente com o biomarcador de peroxidação lipídica. Quando analisado esse fator, verificou-se que resultados favoráveis coincidiram com tempo superior a 7 dias de suplementação ${ }^{33,34,38,39}$, sendo 8 semanas o mais longo período de suplementação encontrado ${ }^{40}$.

Neste estudo, a dieta foi administrada por ao menos 5 dias, porém foram necessários 3 dias para completar as necessidades calóricas. Dessa forma, os pacientes receberam o VET total por pelo menos 2 dias. O tempo de suplementação foi menor quando comparado aos demais estudos, podendo-se supor que esse seja um dos fatores que interferiram na modificação dos níveis de biomarcadores, porém optou-se por realizar a segunda coleta após esse período de tempo devido à alta incidência de óbitos.

A evolução do estresse oxidativo ocorre em pacientes críticos, devido à redução da atividade de antioxidantes endógenos ${ }^{41}$. Segundo o estudo de Bonithon-Kopp et al. ${ }^{42}$, os níveis de MDA foram significativamente mais altos em homens com placas na carótida quando compa- 
rados com os sem placas, denotando estresse oxidativo em doentes crônicos. Pesquisas ${ }^{43-45}$ demonstram que a oxidação de lipídeos, ácidos nucleicos ou proteínas teoricamente podem contribuir para o desenvolvimento de doenças crônicas. Segundo esse autor, embora a suplementação de vitamina $C$, vitamina $E$ e selênio tenha mostrado uma redução na concentração de biomarcadores associados ao estresse oxidativo, a relação entre tais observações e doenças crônicas merece aprofundamento por meio de novos estudos. Sugere-se, portanto, que no caso do AVE, que ocorre com maior frequência em pacientes com doença crônica subjacente, o estresse oxidativo seja tão exacerbado que a ação de antioxidantes alimentares pode não ser suficiente para sobrepor o grau de estresse existente. Além disso, neste estudo não houve possibilidade de comparação com um grupo que não recebeu antioxidantes dietéticos, já que todas as dietas enterais comercializadas atualmente contêm acréscimo de nutrientes antioxidantes. Assim, não foi possível avaliar se a dieta com antioxidantes evitou um aumento mais acentuado do estresse oxidativo e de suas consequências clínicas.

\section{O N CLUSÃ O}

A dieta enteral industrializada contendo antioxidantes não mostrou modificações no estresse oxidativo proteico e na peroxidação lipídica demonstrada por alterações nos níveis de tióis e MDA plasmáticos, tendo ocorrido aumento da formação da carbonila.

Sugere-se que o tempo de administração da dieta, o estabelecimento de um grupo-controle e das quantidades de antioxidantes a serem ofertadas aos pacientes sejam considerados no delineamento de novos estudos.

\section{A GRADECIMENTOS}

Ao Laboratório de Análises Clínicas do Hospital Santa Casa de Misericórdia de Curitiba pelo auxílio na coleta de plasma, e ao Laboratório de Análises Clínicas da Faculdade de Medicina de Ribeirão Preto da Universidade de São Paulo pela dosagem de MDA plasmático das amostras.

\section{COLABORADORES}

LS YASSIN participou da coleta de dados, da realização de dosagens, da análise de dados e da redação do manuscrito. C FUKUI participou da coleta de dados, da realização de dosagens e da redação do manuscrito. M OLANDOSKI foi responsável pela estatística e participou da análise de dados e da redação do manuscrito. PR AVELES colaborou na realização de dosagens, análise de dados e na redação do manuscrito. SC PINTO e PCD PEREIRA participaram da análise de dados e da redação do manuscrito. C RESNAUER participou da concepção do projeto, análise de dados e da redação do manuscrito. LS NAKAO e II MORIMOTO participaram da concepção do projeto, da realização de dosagens, da análise de dados e da redação do manuscrito.

\section{REFERÊ NCIAS}

1. Brasil. Ministério da Saúde. Secretaria de Gestão Estratégica e Participativa. Painel de indicadores do SUS n.1. Brasília: MS; 2006 [acesso 2007 mar 22]. Disponível em: <http://www.rebidia.org.br/ imprensa2/noticias/painel_indicadores_do_ sus.pdf>.

2. Leinonen JS, Ahonen JP, Lönnrot K, Jehkonen $M$, Dastidar P, Molnár G, et al. Low plasma antioxidant activity is associated with high lesion volume and neurological impairment in stroke. Stroke. 2000; 31:33-9.

3. Ferreira ALA, Matsubara LS. Radicais livres: conceitos, doenças relacionadas, sistema de defesa e estresse oxidativo. Rev Ass Med Bras. 1997; 43(1): 61-8.

4. Alexandrova ML, Bochev PG. Oxidative stress during the chronic phase after stroke. Radic Biol Med. 2005; 39(3):297-316. doi: 10.1016/j.freerad biomed. 2005.04.017.

5. Margaill I, Plotkine M, Lerouet D. Antioxidant strategies in the treatment of stroke. Free Radic Biol Med. 2005 15;39(4):429-43. doi: 10.1016/j. freerad biomed.2005.05.003.

6. Acworth IN, Oxon P, Phil D. The handbook of redox biochemistry. Chelmsford; 2003.

7. Moran LK, Gutteridge JMC, Quinlan GJ. Thiols in cellular redox signalling and control. Rev Curr Med Chem. 2001; 8(7):763-72. 
8. Bianchi MLP, Antunes LMG. Radicais livres e os principais antioxidantes da dieta. Rev Nutr. 1999; 12(2):123-30. doi: 10.1590/S114-5273199900020 0001.

9. Cherubini A, Polidori MC, Bregnocchi M, Pezzuto $\mathrm{S}$, Cecchetti R, Ingegni T, et al. Antioxidant profile and early outcome in stroke patients. Stroke. 2000; 31(10):2295-300.

10. Soneghet RM, Piovacari SMF, Oliveira RMC. Disfagia: avaliação e tratamento. In: Knobel E, Oliveira RMC, Cal RGR. Nutrição em Terapia Intensiva. São Paulo: Atheneu; 2005.

11. Guyton AC, Hall JE. Tratado de fisiologia médica. Rio de Janeiro: Elsevier; 2006.

12. Food Trial Collaboration. Poor nutritional status on admission predicts poor outcomes after stroke: observational data from the food trial. Stroke. 2003; 34:1450-6.

13. Kreymann KG, Berger MM, Dutz NED, Hiesmayr $M$, Jolliet P, Kazandjiez G, et al. ESPEN Guidelines on Enteral Nutrition Intensive Care. Clin Nutr. 2006; 25(2):210-23. doi: 10.1016/j.clnu.2006.01.021.

14. Harris JA, Benedict FG. A biometric study of human basal metabolism. Communicated October 8th of the Canegie Institute of Washington; 1918.

15. Long CL, Schaffel N, Geiger JW. Metabolic response to injury and illness: estimation of energy and protein needs from indirect calorimetry and nitrogen balance. J Parent Ent Nutr. 1979; 3(6):452-56.

16. Himmelfarb J, McMonagle E. Albumin is the major plasma protein target of oxidant stress in uremia. Kidney Int. 2001; 60:358-63.

17. Buege JA, Aust SD. Microssomal lipid peroxidation. Methods Enzymol. 1978; 52:302-10.

18. Bamford J, Dennis M, Sandercock P. The frequency, causes and timing of death within 30 days of a first stroke: the Oxfordshire community stroke project. J Neurol Neurosurg Psychiatry. 1990; 53(10): 824-9.

19. Madamanchi NR, Hakim ZS, Runge MS. Oxidative stress in atherogenesis and arterial thrombosis: the disconnect between cellular studies and clinical outcomes. J Thromb Haemost. 2005; 3(2):254-67. doi: 10.1111/j.1538-7836.2004.01085.x.

20. Poulet R. Acute hypertension induces oxidative stress in brain tissues. J Cereb Blood Flow Metab. 2006; 26(2):253-62. doi: 10.1038/sj.jcbfm.96001 88.

21. Sociedade Brasileira de Doenças Cerebrovasculares. Primeiro consenso brasileiro para trombólise no acidente vascular cerebral isquêmico agudo. Arq Neuropsiquiatr. 2002; 60(3-A):675-80.

22. Chang CY, Lai YC, Cheng TJ, Lau MT, Hu ML. Plasma levels of antioxidant vitamins, selenium, total sulfhydryl groups and oxidative products in ischemic-stroke patients as compared to matched controls in Taiwan. Free Radic Res. 1998; 28(1):15-24.

23. Williams RH, Maggiore JA, Reynolds RD, Helgason CM. Novel approach for determination of the redox status of homocysteine and other aminothiols in plasma from healthy subjects and patients with ischemic stroke. Clin Chem. 2001; 47:1031-9.

24. Andersson A, Hultberg B, Lindgren A. Redox status of plasma homocysteine and other plasma thiols in stroke patients. Atherosclerosis. 2000; 151:515-39.

25. Jones DP, Mody VC Jr, Lynn MJ, Stemberg P Jr. Redox analysis of human plasma allows separation of pro-oxidant events of aging from decline in antioxidant defenses. Free Radic Biol Med. 2002; 33:1290-300.

26. Droge W. Aging-related changes in the thiol/ disulfide redox state: implications for the use of thiol antioxidants. Exp Gerontol. 2002; 37:1333-45.

27. Nelson JL, DeMichele SJ, Pacht ER, Wennberg AK. Effect of enteral feeding with eicosapentaenoic acid, gama-linolenic acid, and antioxidants on antioxidant status in patients with acute respiratory distress syndrome. JPEN J Parenter Enteral Nutr. 2003; 27(2):98-104.

28. Betters JL, Criswell DS, Shanely RA, van Gammeren D, Falk D, Deruisseau KC, et al. Trolox attenuates mechanical ventilation-induced diaphragmatic dysfunction and proteolysis. Am J Respir Crit Car Med. 2004; 170(11):1179-84.

29. Zergeroglu MA. Mechanical ventilation-induced oxidative stress in the diaphragm. J Appl Physiol. 2003; 95(3):1116-24.

30. Polidori MC, Cherubini A, Stahl W, Senin U, Sies H, Mecocci P. Plasma carotenoid and malondialdehyde levels in ischemic stroke patients: relationship to early outcome. Free Radic Res. 2002; 36(3):265-8.

31. El Kossi MMH, Zakhary MM. Oxidative stress in the context of acute cerebrovascular stroke. Stroke. 2000; 31:1889-92.

32. Preiser JC, Gossum AV, Berré J, Vincent JL, Carpentier $Y$. Enteral feeding with a solution enriched with antioxidant vitamins $A, C$, and $E$ enhances the resistance to oxidative stress. Crit Care Med. 2000; 28(12):3828-32.

33. Crimi E, Liguori A, Condorelli M, Cioffi M, Astuto $\mathrm{M}$, Bontempo P. The beneficial effects of antioxidant supplementation in enteral feeding in critically III patients: a prospective, randomized, double-blind, placebo-controlled trial. Anesth Analg. 2004; 99(3): 857-63.

34. Institute of Medicine. Dietary reference intakes for vitamin A, vitamin K, arsenic, boron, chromium, copper, iodine, iron, manganese, molybdenum, 
nickel, silicon, vanadium and zinc. Washington (DC): The National Academy Press; 2001.

35. Institute of Medicine. Dietary reference intakes for vitamin C, vitamin E, selenium, and carotenoids. Washington (DC): The National Academy Press; 2001.

36. Abilés J, Cruz AP de la, Castaño J, Rodríguez-Elvira M, Aguayo E, Moreno-Torres R, et al. Oxidative stress is increased in critically ill patients according to antioxidant vitamins intake, independent of severity: a cohort study. Crit Care. 2006, 10(5): R146. doi: 10.1186/cc5068.

37. Ullegaddi R, Powers HJ, Gariballa SE. Antioxidant supplementation enhances antioxidant capacity and mitigates oxidative damage following acute ischaemic stroke. Eur J Clin Nutr. 2005; 59(12): 1367-73. doi: 10.1038/sj.ejcn. 1602248.

38. Elmafda I, Rust P, Majchrzak D, Wagner KH, Genser $D$, Lettner $R$, et al. Effects of beta-carotene supplementation on free radical mechanism in healthy adult subjects. Int I Vitam Nutr Res. 2004; 74(2):147-52.

39. Briviba K, Schnabele K, Rechkemmer G, Bub A. Supplementation of a diet low in carotenoids with tomato or carrot juice does not affect lipid peroxidation in plasma and feces of healthy men. J Nutr. 2004; 134(5):1081-3.
40. Kim JY, Paik JK, Kim OY, Park HW, Lee JH, Jang Y, et al. Effects of lycopene supplementation on oxidative stress and markers of endothelial function in healthy men. Atherosclerosis. 2011; 215(1):189-95.

41. Lamy M, Deby-Dupont G. Oxidative stress. Euroanesthesia. 2005; 12(7):261-73.

42. Bonithon-Kopp C, Coudray C, Berr C, Touboul PJ, Fève JM, Favier $A$, et al. Combined effects of lipid peroxidation and antioxidant status on carotid atherosclerosis in a population aged 59-71 y: The EVA Study 1. Am J Clin Nutr. 1997; 65(1):121-7.

43. Mayne ST. Antioxidant nutrients and chronic disease: use of biomarkers of exposure and oxidative stress status in epidemiologic research. J Nutr. 2003; 133(Suppl 3):933S-40S.

44. Yang M, Chung SJ, Chung CE, Kim DO, Song WO, Koo SI, et al. Estimation of total antioxidant capacity from diet and supplements in US adults. Br J Nutr. 2011; 15:1-11.

45. Jerome-Morais A, Diamond AM, Wright ME. Dietary supplements and human health: for better or for worse? Mol Nutr Food Res. 2011; 55(1): 122-35. doi: 10.1002/mnfr.201000415.

Recebido em: 27/5/2011

Versão final em: 24/1/2012

Aprovado em: 23/3/2012 
